\title{
Mba'e reko ykeregua? Algunas consideraciones sobre aislamiento social y discapacidad en Argentina
}

\section{Mba'e reko ykeregua? Some considerations on social isolation and disability in Argentina}

\author{
Lelia Schewe ${ }^{a}$
}

\begin{abstract}
Resumen
El COVID-19 causó profundas reorganizaciones en las dinámicas sociales de nuestros países. Algunos trabajos sobre colectivos vulnerados han manifestado que el aislamiento social ya estaba instalado antes de la necesidad de ser preventivo y obligatorio, porque se construyeron prácticas violentas, que actúan como motivos de exclusión.

Entre esos colectivos, las personas con discapacidad, han sido obligadas a aislarse por sus discapacidades o bien, han tenido que permanecer separadas, porque las barreras existentes en sus entornos inmediatos no les permiten desplazarse, para construir una vida plena, autónoma, con participación en las comunidades.

En este ensayo se pretende comunicar algunas experiencias en el contexto de nuestra vecindad transfronteriza, desde la provincia de Misiones y las reflexiones que comenzamos a tejer desde el compromiso social de los investigadores de varias universidades de Argentina para superar esos otros aislamientos.
\end{abstract}

Palabras clave: aislamiento social, pandemia, discapacidad, Argentina, COVID-19.

\footnotetext{
Abstract

COVID-19 caused profound reorganizations in the social dynamics of our countries. Some studies on vulnerable groups have shown that social isolation was already installed before the need to be preventive and mandatory, because violent practices were built, which act as reasons for exclusion.

Among these groups, people with disabilities have been forced to isolate themselves due to their disabilities or they have had to remain separate, because the existing barriers in their immediate surroundings do not allow them to move, to build a full, autonomous life, with participation in the communities.

This essay aims to communicate some experiences in the context of our cross-border neighborhood, from the province of Misiones and
}

\author{
${ }^{a}$ Universidad Nacional \\ de Misiones, Instituto de \\ Estudios Sociales y Humanos \\ (IESyH- UNaM-CONICET), \\ Argentina. \\ Correspondencia a: \\ lelia.schewe@gmail.com \\ Recibido: \\ 2o de mayo de 2020 \\ Aceptado: \\ 29 de junio de 2020 \\ Artículo publicado en acceso \\ abierto bajo la Licencia \\ Creative Commons.

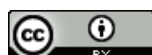 \\ Cita: \\ Schewe, L. (2020). Mba'e \\ reko ykeregua? Algunas \\ consideraciones sobre \\ aislamiento social y \\ discapacidad en Argentina. \\ Kera Yvoty: reflexiones sobre \\ la cuestión social, 5(número \\ especial), 30-35.
}


the reflections that we began to weave from the social commitment of researchers from various universities in Argentina to overcome these other isolations.

Keywords: social isolation, pandemic, disability, Argentina, COVID-19.

\section{Introducción}

El COVID-19 causó profundas reorganizaciones en las dinámicas sociales de nuestros países. Algunos trabajos sobre colectivos vulnerados han manifestado que el aislamiento social ya estaba instalado antes de la necesidad de ser preventivo y obligatorio, porque hay prácticas violentas, que actúan como motivos de exclusión.

Entre esos colectivos, las personas con discapacidad, han sido obligadas a aislarse por sus discapacidades o bien, han tenido que permanecer separadas porque las barreras existentes en sus entornos inmediatos, no les permiten desplazarse, para construir una vida plena, autónoma, con participación en las comunidades.

Como desplazarse no es la única forma de vincularse, también es necesario mencionar que, las alternativas virtuales para las continuidades durante la pandemia del COVID-19, colaboran con las exclusiones y el aislamiento en la medida en que las personas con discapacidad no han recibido en su acceso a la educación las herramientas para comprender y utilizar esos medios, los espacios virtuales no cumplen con las condiciones de accesibilidad o bien, no tienen teléfonos y/o computadoras.

En este ensayo se pretende comunicar algunas experiencias en el contexto de nuestra vecindad transfronteriza, desde la provincia de Misiones y las reflexiones que comenzamos a tejer desde el compromiso social de los investigadores de varias universidades de Argentina. La intención de compartir este trabajo se vincula también a los diálogos que se han construido en nuestras fronteras. Sabemos que tanto las lenguas, como las costumbres y las dinámicas sociales no son privativas de cada país, porque las fronteras se permean y dan lugar a creaciones nuevas, trans-fonterizas. En un trabajo anterior (Schewe, 2020) proponíamos un diálogo ente lenguas, porque, en las fronteras en contacto, se pregunta en un idioma y se contesta en otro, como forma de comunicación. Con las temáticas vinculadas a los temas que se abordan aquí, también encontramos diálogos, sobre todo cuando, por ejemplo, entre Posadas y Encarnación cruzamos el puente para intercambios profesionales, atención de las personas con discapacidad, capacitación y formación.

\section{En aislamiento involuntario}

Una llamada telefónica, a 6o días de aislamiento social preventivo y obligatorio, me conmovió. Era un estudiante con discapacidad intelectual que cursa el tercer año de una carrera de formación docente en la universidad donde trabajo, diciéndome:

"Profe, mis compañeras están hablando de una plataforma, que hay que anotarse, donde los profes van a hablarnos, ¿usted sabe algo de eso? ¿Dónde es esa plataforma?"

Traté de explicar con claridad, respondiendo a las preguntas, pero me sorprendió aún más la tercera:

“Eso es por internet?”

Primero, cuando empecé a indicar las cuestiones hablando de páginas Web, aulas virtuales y asignaturas universitarias, no se me había ocurrido mencionar la conectividad, daba por obvio que el estudiante sabía que estábamos hablando de eso. En segundo lugar, me di cuenta que yo misma había naturalizado esa figura del estudiante ideal, que tiene un teléfono celular de último modelo, que puede pagar una conexión a internet y que ya usó en algún momento de su escolaridad, una "plataforma virtual".

La situación me llevó a varias exploraciones que aparecen en estas 
páginas, develando que muchos de nosotros incorporamos las nuevas dinámicas virtuales muy rápidamente, causándonos estados de hiperconexión, precarizando nuestras vidas aún más que en la presencialidad, pero ¿qué pasa con quienes no cuentan con esos medios? Si para las continuidades en aislamiento por la pandemia necesitamos estar conectados a internet, ¿cómo saldaremos las discontinuidades?

Varios autores (Eraso \& Andreu, 2017; Morales \& Rotela, 2020; Prieto et al., 2018; Rojas, 2016) han vinculado las implicancias de los aislamientos a sus estudios sobre la discapacidad. En nuestra experiencia, el relato de una entrevistada en un proyecto de investigación, también resultó revelador: en un espacio de militancia contra las violencias hacia mujeres con discapacidad habían escuchado la historia de una mujer de más de 20 años a quien sus padres no le permitían salir de su casa: solamente había ido a visitar al médico con su madre. Las integrantes del espacio de militancia se acercaron a la casa de la familia y hablaron con los padres, quienes con total naturalidad afirmaban que era imposible que saliera, porque las personas que denominaban inválidas, no sobrevivirían en la calle, menos aún si son mujeres. Lo curioso es que, lo manifestaban teniendo frente a ellos un grupo de 5 mujeres con discapacidad visual que habían viajado durante horas a un lugar que no conocían, a buscar a su hija.

Como el caso del estudiante mencionado anteriormente, esta situación tampoco forma parte de las estadísticas oficiales: las mujeres decidieron no denunciar para poder acompañar el caso y lograron que la mujer que había sido encerrada toda su vida, pueda asistir a los talleres que dictan, aprender a leer y a escribir, aprender sobre educación sexual. Con respecto a la situación anterior, al comenzar el intento de educación en la virtualidad, se realizó una encuesta sobre las condiciones de acceso a internet de los estudiantes: los resultados que arrojó, evidenciaban que casi el $100 \%$ de quienes se sumarían a la propuesta tenían celular (y/o computadoras) y acceso a internet. ¿Por qué medio se realizó la encuesta? ¡Por correo electrónico!

Si suponemos que las personas con discapacidad si tienen acceso a internet, podríamos entender que una parte del problema estaría resuelto. Pero, un informe que armamos con un equipo de la Universidad de Quilmes (Argentina), sobre accesibilidad Web (Toledo et al., 2019) demuestra que no es tan fácil determinar si una persona con discapacidad puede usar cómodamente una página de internet. Aunque existen programas automáticos que evalúan los indicadores, es necesario que sean los usuarios quienes determinen, con el uso, si son adecuados. Afirmábamos, en el informe, que "para lograr que los desarrollos tecnológicos y la información digital [se] planteen el menor número posible de obstáculos para cualquier usuario, es necesaria la aplicación de los principios de accesibilidad, usabilidad, interoperabilidad y/o del Diseño Universal. A partir de la aplicación de la concepción del Diseño Universal, el desarrollo de los productos digitales se realiza desde un punto de vista ecológico, es decir, considerando las necesidades e intereses de todos los posibles usuarios" (2019, p. 34). Lo mismo pasa con cada uno de los apoyos específicos que se construyan: por eso es necesaria la participación activa de las personas con discapacidad.

Una compañera investigadora con discapacidad visual, nos compartía, por ejemplo, que cuando cursaba asignaturas de grado, la forma de que acceda a los exámenes que estaba al alcance de los profesores era que ella haga sus exámenes de forma oral. Su problema con eso estaba vinculado a su timidez, así que le costaba bastante presentarse a rendir y le causaba mucho estrés, por lo que a veces decidía faltar, aunque eso implicaba que su carrera le llevara mucho más tiempo que a sus compañeros sin discapacidad. En este caso, las propuestas virtuales implican fotografías, ser parte de videos 
sin consentimiento, compartir datos personales, números telefónicos, entre varias otras cuestiones que generan sobreexposición y exposición, muchas veces, no contemplados en las propuestas y sin consultar a los participantes, ¿cuáles serían las alternativas en los casos de incomodidad ante lo virtual, para que no signifiquen destiempos?

En estos tiempos de COVID-19, las exigencias de conexión proponen otras incomodidades, que se transforman en nuevasymásacentuadasformasdeviolencias y exclusión. Como habíamos explorado en otro trabajo (Schewe \& Blogna, 2020), somos conscientes de que en los últimos años se han ido construyendo prácticas que colaboran con formas de participación de las personas con discapacidad que resultan interesantes, pero, aún persisten respuestas incoherentes, insuficientes.

Por eso, es relevante en campo de saberes de los Estudios Críticos en Discapacidad, el planteo de Mello y Mozzi (2018) sobre la dimensión política del cuidado y del dolor. Las autoras sostienen que es necesario incluir al capacitismo, como forma de violencia específica, en los estudios interseccionales vinculados a las teorías feministas. Lo definen como una forma de jerarquización de las personas en función de la adaptación de sus cuerpos a la hegemonía de la corponormatividad. En este caso, en tiempos de COVID-19, imaginamos y naturalizamos cuerpos que operan con computadoras y celulares, que se comunican sin apoyos, que forman parte de las dinámicas de las redes sociales, hasta podemos decir que nos representamos seres que tienen ciertas formas estéticas. Lo preocupante no es que los naturalicemos, sino que dirigimos la mayoría de las propuestas que generamos a esa supuesta generalidad, a la que, si observamos con cuidado, es necesario adaptarse. Esto también implica que fomentamos la homogeneidad, a veces, sin pensarlo, bajo formas políticamente correctas y buenas intenciones.

Otra característica de las dinámicas de esta época de pandemia es el estado de angustia que genera la incertidumbre. En algunos casos, en respuesta a los planteos sobre esas emociones, aparecen, entre otras, "angustia es tener hambre" o "no pueden angustiarse quienes tienen un techo para refugiarse". En este caso, es necesario recordar lo que causa la desinformación: entre las dinámicas de intercambio en nuestras investigaciones, nos encontramos con personas con discapacidad que manifiestan estar asustadas, porque los medios de comunicación a los que acceden pregonan muerte y desesperación. Por ejemplo, ¿qué sentidos le atribuye una persona que no accede a información clara y accesible a una imagen de cientos de ataúdes en fosas comunes?

\section{Cuando la coyuntura no permite conclusiones: propuestas y perspectivas}

Parece desalentador que estemos hablando (escribiendo, en este caso) aún sobre confinamientos por motivos de discapacidad. Entendemos que "el caso de Paraguay no fue diferente, la discapacidad se mantuvo mucho tiempo invisible a la vida pública, solo enmarcada en el ámbito doméstico, y por lo tanto asumida y resuelta por este ámbito" (Ruffinelli \& García, 2018, p. 32), por lo que una de las perspectivas podría tratarse de sacar del ámbito de lo privado las discusiones sobre las formas de resolver las exclusiones. En este caso, en pandemia, el trascender ese espacio tiene también esta forma, de escritura, en medios de confianza para leernos y escribirnos.

De la misma manera que en Argentina, entendemos que, en Paraguay, varios colectivos se encuentran en lucha por el cumplimiento de los compromisos estatales con respecto a las personas con discapacidad, como el cumplimiento de la Ley 5136 (Velázquez, 2020). Otra de las propuestas se trata de contemplar las complejidades de esta situación y aclarar cuáles serían las nuevas maneras de construir esos nuevos y necesarios entornos accesibles para la participación. 
Una recomendación de la Comisión Económica para América Latina y el Caribe (CEPAL) dice que es una necesidad imperante: "asegurar la accesibilidad a toda la información relacionada con la crisis. Esto incluye la información de salud pública para prevenir, detectar y tratar el COVID-19 e información sobre las medidas implementadas por los gobiernos para enfrentar y mitigar los impactos socioeconómicos de la pandemia, de modo que las personas con discapacidad pueden estar informadas sobre prestaciones a las que puedan acceder en el contexto de la crisis y en el período de recuperación. Esta información debe estar disponible en lenguaje de señas y en lenguaje sencillo y fácil de leer, en medios, modos y formatos accesibles, lo que incluye la tecnología digital, los subtítulos, los servicios de retransmisión y los mensajes de texto" (CEPAL, 2020, p. 3). Ya se mencionaron aquí las dificultades que esto implica en cuanto a recursos, pero se trata de acciones fundamentales para garantizar el acceso de las personas con discapacidad.

Una de las urgencias: las mujeres con discapacidad que están aisladas e incomunicadas y son víctimas de violencias, están cada vez más expuestas a las más crueles vejaciones, que, en este contexto de aislarnos para cuidarnos, contradictoriamente, le da más impunidad a los abusadores. En este caso, recurrimos a la figura del vecino del título de este trabajo, no solamente para referirnos al vecino país, sino también porque una de las formas de colaborar con el flagelo de la violencia contra las mujeres, es estar atentos, ocuparse y denunciar.

Con respecto a la salud, la prioridad en este momento es el COVID-19, pero, sabemos que es necesario sostener acciones para colaborar con la salud mental, en general y en particular de quienes presentan vulnerabilidades que requieren otras formas de atención y asistencia. Echevarría et al. (2020), desde las condiciones de Perú, se ocuparon de señalar, que las estrategias pueden ser variables en el tiempo y precisan de seguimiento y evaluación permanente, es necesario recordar esta cuestión porque hay acompañamientos profesionales que están siendo imposibilitados de continuar virtualmente y que las rutinas cambiantes podrían causar profundos sufrimientos.

En otra dimensión que ocupó gran parte de este artículo, la educativa, un postulado comienza a oírse (y a leerse), mientras se discute aún si estamos en tiempos de continuidad pedagógica, interrupción de la escolaridad o creación de estrategias: eso que estamos tratando de hacer no es educación virtual, la educación (virtual o no) será con todas/os/es los estudiantes.

\section{Referencias}

Comisión Económica para América Latina y el Caribe (CEPAL) (2020). Personas con discapacidad ante la enfermedad por coronavirus (COVID-19) en América Latina y el Caribe: situación y orientaciones. Naciones Unidas: CEPAL. https:// repositorio.cepal.org/bitstream/ handle/11362/45491/S2000300_ es.pdf? sequence $=1 \& i$ Allowed $=y$

Echavarría-Ramírez, L., Díaz-Reyes, D. V., \& Narzisi, A. (2020). Trastorno del espectro autista: pautas para el manejo durante el periodo de aislamiento social por el coronavirus. Cuadernos de Neuropsicología/Panamerican Journal of Neuropsychology, 14(1). http:// www.cnps.cl/index.php/cnps/article/ view/397

Eraso, A. B., \& Andreu, X. P. (2017). La exclusión social y sus determinantes relacionados con la salud y la discapacidad. Áreas. Revista Internacional de Ciencias Sociales, (36), 183-196. https://revistas.um.es/ areas/article/view/308221

Mello, A., \& Mozzi, G. (2018). A favor da deficiência nos estudos interseccionais de matriz feminista. En Freitas Rosa, et al. (Ed.), Políticas públicas, relações de gênero, diversidade sexual e raça na perspectiva interseccional. Secco Editora. 
Morales, L. N., \& Rotela, C. A. (2020). Tipos de discapacidad en una comunidad de Caazapá. Anales de la Facultad de Ciencias Médicas, 52(3), 69-76). https://revistascientificas.una.py/ index.php/RP/article/view/1639

Prieto, D., Díaz, F., Astorga, Y., \& Estay, J. (2018). Psicosis, Discapacidad intelectual y trastornos conductuales: enfoque biopsicosocial. Psiquiatría y Salud Mental, 35(3/4), 262-267. Recuperado a partir de http://www. schilesaludmental.cl/wp-content/ uploads/2019/05/18.-psicosis discapacidad-intelectual-y-tto.conductuales-D.-Prieto-y-cols.pdf

Rojas, G. A. S. (2016). De la anomalía a la discapacidad, una larga historia de exclusión social: de la muerte, al destierro y el repudio, a la inclusión educativa (From Anomaly to disability, a long history of social exclusion). Inclusión $\mathcal{E}$ desarrollo, 3(2), 34-46. https://revistas.uniminuto.edu/ index.php/IYD/article/view/1270

Ruffinelli, R., \& García, S. (2019). Políticas públicas y discapacidad en el Paraguay. Revista Científica Estudios e Investigaciones, 7, 32-33. https://doi. org/10.26885/rcei.foro.2018.32
Schewe, L. (2020). "As deusas nos protejam dessas novas cruzadas". Anahí Guedes de Mello, anticapacitismo feminista desde el sur global. Revista Nómadas, 52.

Schewe, L., \& Blogna, S. (2020). Políticas públicas y discapacidad: ilegalidad(es) y disputas actuales. En R. Baquero, P. Scharagrodsky, \& S. Porro (Eds.), Discursos, prácticas e instituciones educativas. Buenos Aires: Prometeo.

Toledo, G., Aguerrido, M., Rapanelli, A., Zorz, S., Gallardo, H., Canto, S., Pignataro, M., Schewe, L., \& Fernandez, A. (2019). Personas con discapacidad yel acceso a los servicios de la información y la comunicación. Recomendaciones a partir de un estudio exploratorio realizado en la región del cono sur. Bernal: UNQ. https://accesibilidaddigital.adc.org.ar/index.html

Velázquez, L. (2020). La educación inclusiva de las personas con discapacidad visual en tiempos de pandemia en Paraguay. http://periodicodiscalupa. sintoniadeinclusion.com/articulo. ph p / educacion-inclusiva/laeducacion-inclusiva-de-las-personascon-discapacidad-visual-en-tiemposde-pandemia-en-paraguay $/$ ?id=214

\section{Sobre la Autora}

\section{Lelia Schewe}

Docente investigadora de la Universidad Nacional de Misiones, Instituto de Estudios Sociales y Humanos (IESyH- UNaM-CONICET). Profesora en Educación Especial, Magister en Ciencias Sociales y Humanidades (UNQ) y doctoranda en Ciencias de la Educación (UNLP). 